\title{
BLADE CUP METHOD FOR CAVITATION REDUCTION IN MARINE PROPELLERS
}

\author{
M. Burak Samsul \\ Istanbul Technical University, Türkiye \\ * Corresponding author: samsul@itu.edu.tr (M.B. Samsul)
}

\begin{abstract}
Energy efficiency has become more important in every industry and daily life. Designing and building a more efficient marine vehicle can lead to lower fuel consumption and a longer lifetime for the components of the vehicle.

Erosion caused by cavitation reduces the service life of the propeller and the related components in the propulsion and maneuvering system. Reducing cavitation leads to a longer life for these components. This paper aims to explain and investigate propeller blade cup as a cavitation reduction method for marine propellers. A cavitating no-cup propeller is created and analyzed then the cupped version of this propeller is generated and analyzed to compare with the no-cup propeller. Cavitation results of these propellers are investigated. In addition, the thrust, torque, and efficiency of the propellers are compared.
\end{abstract}

Keywords: Marine Propeller,Computational Fluid Dynamics,Propeller Cup,Cupping,Cavitation,Propeller Efficiency,Vibration,Noise,Erosion

\section{INTRODUCTION}

A propeller is expected to create the required thrust to overcome the ship resistance at a specified speed. The main engines supply the torque to the propeller. The torque is limited by the engine power and rotational speed of the propeller. Basic propeller design principles give the designer general ideas for how to design a propeller for a particular vessel. In most cases, the propulsion system and components design are made for building a new ship. A newly designed ship provides great flexibility to the Naval Architect to modify most of the design inputs. Propeller diameter, pitch, and blade area ratio can be determined in the design of the propeller. Also, if the propeller design process is started at the very early stages of the project, engine power and gearbox reduction ratio can be determined more accurately. Even hull stern shape can be optimized because the hull-induced velocity distribution has a critical effect on the propeller [1] [2].

For the propeller design, the most important goals are satisfying the speed and efficiency expectations based on the operating conditions and requirements of the vessel. For some of the design processes on marine propellers, the operating conditions of the propeller may need to be forced to the limits. This can be a necessity for reaching the design goals. Cavitation is a critical design limit for marine propellers. Operating in the cavitating zone leads to noise, vibration, and erosion problems, which cause permanent damage to the propeller and on related components [3] [4]. Also, propeller 
performance is affected by cavitation [5]. Any propeller that suffers from the mentioned problems may not be able to meet the speed and comfort expectations after a while of operation. In addition, propeller replacement may be needed because of the damage caused by erosion on the propeller. Nevertheless, in some cases, propeller design may have more strict limitations and challenges. For instance, the propeller diameter can be limited because of the hull geometry. The shaft angle may create diameter restrictions to the propeller coming from shaft installation restrictions on the boat. In order to meet the speed expectations, the propeller may be forced to work in cavitating conditions. Propeller section modification can be the only option to eliminate the cavitation while satisfying the speed and efficiency requirements if all of the mentioned limitations and difficulties are considered [6].

Blade pitch increment is an option to create more thrust if the diameter is not able to be increased. It is well-known that increased blade pitch increases the likelihood of creating cavitation. Cavitation reduction can be achieved by modifying the pitch distribution on a blade starting from the root to the tip. High loading of the blade tips and unloading the midsections of the blade provide a reduction in sheet cavitation. However, this method leads to increased tip loading and propulsive efficiency loss [7].

In this study, cavitation reduction is achieved by not changing the shaft depth, rotational speed of the propeller, or blade area ratio of the propeller. The only modified parameter is the blade trailing edge shape and angle of attack of the blade. Blade trailing edge deflection, which is called the propeller blade cup, is a beneficial option to modify the pressure distribution on the blade. Also, the propeller cup method provides the opportunity to decrease the angle of attack of the propeller, which has a significant effect on cavitation reduction [6] [8].

For this study, two propellers are created. The first is a no-cupped propeller and the second is the cupped version of the first propeller, which has a decreased angle of attack. Propeller cupping increases the effective pitch [6] [8] [9], so the blade angle of attack is decreased in the cupped propeller in order to have the same effective pitch with the initial no-cup cavitating propeller. These two propellers with the same effective pitch provide the same open water characteristics if the cupping level and angle attack decrement is correctly applied [8] [10]. The specified no cupped conventional propeller is a cavitating propeller. The cupped version of this propeller has a blade cup on the trailing edge, and the angle of attack of the blade is reduced to match the produced thrust [9].

This paper investigates the cavitation reduction effect of the blade cup by the Computational Fluid Dynamics (CFD) solution. In addition, previous studies in this field do not provide a CFD solution that examines how to modify the angle of attack of the blade for cavitation reduction [6] [8] [10] [11]. Cupping is an effective solution to modify the cavitation characteristic of a propeller without decreasing its efficiency. The study shows that the cupping application provides a good opportunity to modify the peak low pressure values on the blade.

\section{CAVITATION}

Cavitation is a fluid mechanics phenomenon that can occur whenever a liquid is used in a machine, inducing pressure and velocity fluctuations in the fluid. Where the pressure is significantly low due to the high local velocities, the fluid phase changes from liquid to vapor, which causes the cavitation to occur.

If cavitation inception is investigated at a point $\mathrm{A}$, the local pressure of the point is defined by $\mathrm{Pa}$, then;

$$
P_{A} \leq P_{V} ; \frac{P_{0}-P_{A}}{\frac{1}{2} \rho V^{2}} \geq \frac{P_{0}-P_{V}}{\frac{1}{2} \rho V^{2}}
$$

where $\mathrm{P}_{\mathrm{A}}$ and $\mathrm{V}_{\mathrm{A}}$ are local flow pressure and velocity, Po and $\mathrm{V}$ are the fluid pressure and velocity at the far upstream flow area where the flow is not affected by the geometry, in the so-called infinity.

By using the Bernoulli equation, a simple criterion for cavitation is defined as the cavitation number. $\sigma$ is the cavitation number and $\mathrm{C}_{\mathrm{p}}$ is the non-dimensional pressure coefficient .

$$
\sigma=\frac{p-p_{V}}{\frac{1}{2} \rho V^{2}}, C_{P}=\frac{P_{0}-P_{A}}{\frac{1}{2} \rho V^{2}} \quad C_{P}=-\frac{\Delta P}{q}
$$

$\sigma \leq \frac{\Delta P}{q} ;$ cavitation occurs,$\quad \sigma \geq \frac{\Delta P}{q} ;$ cavitation does not occur

The types of cavitation depend on the location on the blade of the propeller and the physical appearance itself. Cavitation that depends on the location on the blades are back cavitation and face cavitation. Cavitation that depends on the physical appearance are tip/hub vortex cavitation, sheet cavitation, bubble cavitation, root cavitation, propeller-hull vortex cavitation, and unsteady sheet cavitation. This study mainly examines sheet cavitation and bubble cavitation, with a focus on reducing them on the propeller. These two types of cavitation are generated by low-pressure distribution on the suction side of the blades. Cavitation can occur on a ship component where the local pressures are lower than the evaporation pressure of the water. Because of the creation of high local velocities and low pressures, propellers are one of the main sources of cavitation for marine vehicles.

There are several effects of the cavitation on the propellers, such as performance breakdown, noise, vibration, and erosion. This study mainly focuses on reducing/eliminating blade erosion by reducing the cavitation while keeping the propeller performance constant. 


\section{HOW TO REDUCE CAVITATION}

There are several methods to reduce the cavitation on the propeller, such as decreasing the blade rotational speed, changing the shaft depth, anti-fouling, and pitch reduction.

If the propeller rotation speed is reduced to create a lower local speed distribution around the blade, then the local pressure values are going to be increased. The increment for the local pressures provides an opportunity to create higher pressures than the vapor pressure of the water. Propeller rotational speed reduction, likewise, brings loss in thrust. Enlarging the propeller diameter is an option to gain the lost thrust back; however, physical limits may prevent application of this solution in some cases.

Increasing the depth increases hydrostatic pressure. This means that cavitation occurrence risk is reduced when the depth of the propeller is increased. The depth-increasing method for cavitation reduction is not an applicable solution for the specified cases defined in this paper.

Fouling leads to an increased probability for cavitation and also reduces propeller efficiency [12]. However, if a propeller creates cavitation when it is new, this situation shows that cavitation reduction should be achieved by amending the propeller characteristics. This study focused on the cavitation reduction solutions for the propellers that are already non-fouled.

For both fixed-pitch conventional propellers and controllable pitch propellers, decreasing the angle of attack leads to higher local pressure distribution on the suction side of the blade. Fig. 1 shows a typical pressure distribution on a blade section that has a positive angle of attack.

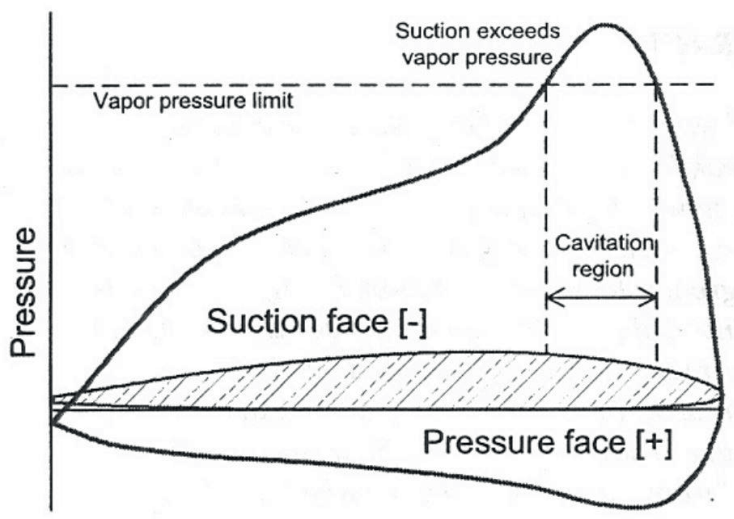

Fig. 1. Typical pressure distribution on a blade section [13]

In order to decrease or eliminate the cavitation, manipulating the pitch by amending the angle of attack is always an option. But pitch decrement is going to bring loss in thrust. If there is no available option to gain back the lost thrust, the pitch reduction application leads to loss in produced thrust.

Modifying the pitch distribution on an existing propeller and creating a new blade that has the same overall pitch but a different angle of attack distribution on the sections of the blade is an option to reduce the cavitation. In addition, cloud cavitation can be prevented or reduced by increasing tip loading and distributing the blade loads more evenly from the maximum pitch region to the tip. The tip loading method to reduce cavitation on the blade provides sheet cavitation reduction. However, high tip loading can also bring up tip vortex cavitation and propulsive efficiency loss from the tip vortex [7].

\section{EFFECT OF ANGLE OF ATTACK ON CAVITATION}

Propellers produce thrust by creating a pressure difference between the suction side and the thrust side of the blades. Increasing the pressure difference between these two faces increases the thrust generated by the propeller. Increasing the angle of attack of the blades, which means increasing the pitch of the propeller, also leads to the generation of a relatively higher pressure difference and thrust. This additional thrust may be needed on a vessel that has requirements and design restrictions, as mentioned in the previous section. However, as shown in Fig. 2, an increased angle of attack leads to cavitation risk on the back (suction side) of the propeller blade.

On the suction side of the blade, especially in the midchord area, the flow velocity is at the highest level, which means that peak low-pressure values are observed in this zone.

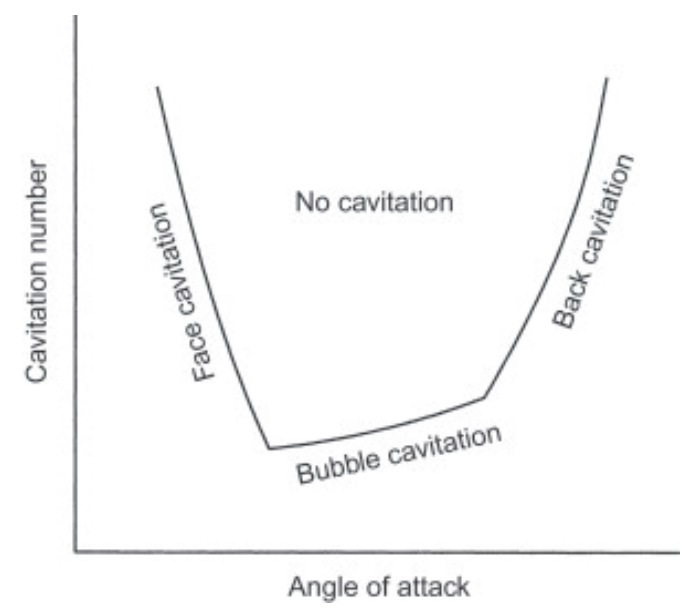

Fig. 2. Angle of attack vs. cavitation number [14]

\section{CUPPING METHODOLOGY}

\section{AIM OF CREATING PROPELLER CUP}

For the specified cases for a boat that are defined in the previous sections, there might be no opportunity to change the propeller characteristics to avoid cavitation. However, cavitation reduction might be needed on these specified challenging cases. In these cases, reducing the rotational speed or angle of attack is going to reduce or eliminate the cavitation as known. But applying these methods leads to thrust and efficiency losses. At this point, the propeller cup 
is very advantageous when the propeller cavitation needs to be reduced while the propeller thrust and efficiency need to be preserved [6].

The purpose of the cupping application on the blades of the propeller is to spread the low pressure on the suction side of the blade to have the pressure distribution above the vapor pressure without changing the total pressure difference between the faces of the blade. With the help of the cup application, local pressure values around the mid-chord are increased where the minimum pressure values are expected to be observed on a conventional propeller. As a result of the blade cup, the local pressure values around the trailing edge of the propeller are decreased compared with a no-cupped conventional blade section [6] [8]. In addition, a blade cup is an anti-singing option. The anti-singing chamfer is typically applied to the suction side of the blade; however, there are some anti-singing edge forms that are applied to both sides [15].

Fig. 3 shows the pressure distribution difference between a conventional blade section and a cupped blade section.

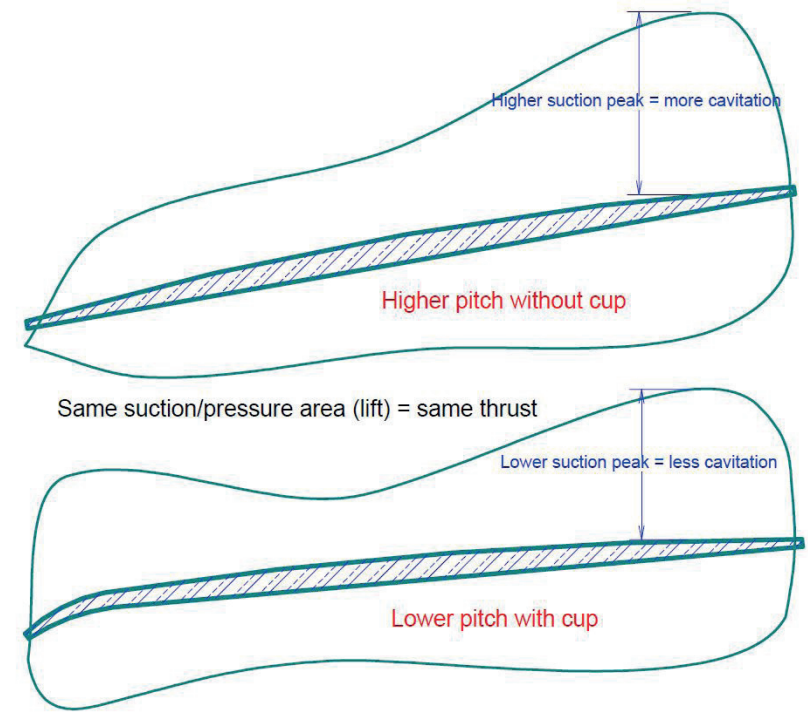

Fig. 3. Pressure distribution on cupped and no-cup blade sections [8]

A propeller cup is simply the deformation of a propeller's trailing edge toward the pressure face. Providing an additional camber to the blade trailing edge changes the pressure distribution along the blade's chord length, adding lift toward the trailing edge. Typically, there is a peak in the lift distribution around the leading half of the blade. This peak lift zone is also the lowest pressure area, in general, for a conventional Fixed Pitch Propeller (FPP). The main purpose is to create a suction side pressure distribution that is above the vapor pressure at the specified condition. It is possible to eliminate/reduce the cavitation, which can be achieved by spreading the lowest pressure values (around the peak lift area on the suction side) to the blade chord. This also means spreading the lift distribution on the chord instead of loading it around the mid-chord zone [7]. Propeller cupping provides an opportunity to modify the pressure distribution on the blade chord. Because effective pitch increases with the effect of cupping, the angle of attack of the blade can be reduced to reach the effective pitch value of the initial no-cup propeller. With the help of this, the lowest pressure value can be changed while keeping the total pressure difference constant, which means keeping the thrust constant.

\section{CUPPING CREATION METHOD}

There is no established and agreed methodology to create a cup on the propeller blades. Different companies make definitions based on their rates. However, the experiments performed at the David W. Taylor Naval Ship Research and Development Center provide extensive data to examine the cupping and cavitation relationship, and also provide a formulation for the cupping vs. effective pitch relationship [6]. In this study, blade cup definitions are made in agreement with Hydrocomp. Fig. 4 shows the propeller diameter /cup drop ratio definition of Hydrocomp [11].

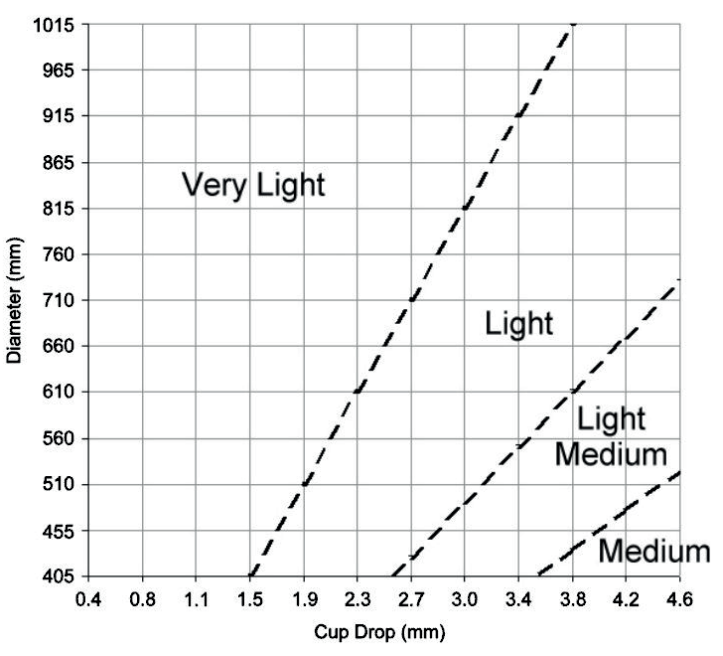

Fig. 4. Propeller diameter vs. cup drop [11]

The total deflection that is given to the trailing edge of the blade is defined as a ratio of the diameters of the propeller. As shown on the cup drop /diameter graph, cup levels are named as very light, light, light medium, medium, medium heavy, and heavy cup. All of these levels are defined as a ratio of propeller diameters. Chord deflection is applied to start from $0.9 \mathrm{C}$ to $1.0 \mathrm{C}$. That means $0-0.9 \mathrm{C}$ of the blade section is the same as the original section. When a chord deflection between $0.9-1.0 \mathrm{C}$ is applied, propeller thrust and torque increased at the same advance coefficient. Then, the blade angle of attack should be decreased on the applied cup version of the propeller to match the effective pitch.

In the following chapters, a cavitating conventional fixedpitch propeller is investigated. Then, that specified propeller is solved after applying the cup and reducing the blade angle of attack. This study provides an opportunity to compare cavitation characteristics of two different propellers that have the same thrust, torque, and efficiency values at the same advance coefficient. 


\section{CAVITATION REDUCTION BY CUP METHOD}

\section{NO CUPPED AND CUPPED PROPELLER COMPARISON}

To observe the cavitation reduction capability of the blade cup, a FPP cavitating propeller is defined by using the lifting surface code. Once the cavitating propeller is obtained and solved at defined advance coefficients, then the cupped version of this propeller is created by following the same procedure. However, the critical point of creating the cupped propeller is to find the angle of attack decrement. The angle of attack optimization can be carried out by two tools. One is the effective pitch-geometric pitch conversion formula, which provides a very basic idea, and the second one is the lifting surface method. The angle of attack decrement for the cupped propeller should be compatible with the applied cupping because the additional torque that is created by the cupping should be adjusted by the angle of attack decrement in order to not exceed the torque of the initial no-cup propeller. After creating the propellers with the help of the lifting surface code, sections of the propeller are converted into a $3 \mathrm{D}$ model.

The camber distribution of the section is selected as NACA66 for the initial conventional propeller. The NACA66 camber is modified to create the determined cup deformation on the blade section. To compare the cavitation characteristics, firstly, the conventional propeller is solved with CFD for two different advance coefficients. The advance coefficients are shown in Table 1.

Tab. 1. Solution advance doefficients, inlet velocities, and propeller revolutions

\begin{tabular}{|c|c|c|}
\hline Advance Coefficient & $\begin{array}{c}\text { Inlet Velocity } \\
(\mathbf{m} / \mathbf{s})\end{array}$ & $\begin{array}{c}\text { Propeller Revolution } \\
\text { (rps) }\end{array}$ \\
\hline 0.82 & 10.28 & 25 \\
\hline 0.87 & 8.7 & 20 \\
\hline
\end{tabular}

Once the thrust and torque values are calculated for the conventional no-cup cavitating propeller, the cupped version of the propeller is created. Several cupping levels are applied on the blade and the angle of attack of the blades is reduced relative to the cupping level of each propeller.. Pitch ratio $(\mathrm{P} / \mathrm{D})$ tuning is done by the lifting surface code. The lifting surface model forms a reliable basis for designing blade section profiles and screw propeller blades [16]. As emphasized, thrust, torque, and efficiency results should be the same as the initial conventional propeller.

There is a practical formulation that proposes a pitch conversion for cupping applications [8] [10]. This formulation does not guarantee that the effective pitch of the propeller can be accurately found. However, the formulation provides an accurate starting point for the CFD calculations. After solving the cupped propeller, which is generated with the help of the formulation, final tuning can be performed with CFD studies.

$$
\mathrm{P}_{\mathrm{EFF}}=\mathrm{P}_{\mathrm{GEO}}+21\left(\mathrm{X}_{\mathrm{CUP}}\right)[8]
$$

where,

$\mathrm{P}_{\mathrm{EFF}}=$ effective pitch

$\mathrm{P}_{\mathrm{GEO}}=$ geometric (uncupped) face pitch

$\mathrm{X}_{\mathrm{CUP}}=$ trailing edge deformation (drop)

Table 2 shows the general characteristics of the created no-cup and cupped propeller. The no-cup propeller has a $675 \mathrm{~mm}$ mean pitch, which is 1.35 in terms of $\mathrm{P} / \mathrm{D}$. After applying the cup to the blades, the cupped propeller mean pitch value should be decreased with the help of the formulation in order to match the effective pitch values of these two propellers. In this case, the trailing edge deformation is $6.2 \mathrm{~mm}$. If a $6.2 \mathrm{~mm}$ cup drop is applied to the blade, which has a $675 \mathrm{~mm}$ mean pitch, the cupped blade mean pitch should be decreased to $675 \mathrm{~mm}-(21 \times 6.2 \mathrm{~mm})=544.8 \mathrm{~mm}$. In this case, the cupped propeller $\mathrm{P} / \mathrm{D}$ is determined to be 1.1 , which is equal to $550 \mathrm{~mm}$.

As emphasized, the blade angle of attack of the cupped propeller should be checked with CFD, and then, final tuning of the cup drop can be achieved.

Tab. 2. No-cup and cupped propeller general characteristics

\begin{tabular}{|c|c|}
\hline \multicolumn{2}{|c|}{ No-Cup and Cupped Propeller } \\
\hline Diameter (mm) & 500 \\
\hline No-Cup P/D & 1.35 \\
\hline Cupped P/D & 1.1 \\
\hline Number of Blades & 3 \\
\hline Expanded Area Ratio & 0.6 \\
\hline Hub Diameter (mm) & 100 \\
\hline Camber & NACA66 \\
\hline
\end{tabular}

The sections of the no-cup and cupped propeller are created according to the values shown in Table 3 . The pitch values on the table show the blade section pitches. The effective pitch of the cupped propeller is the same as the initial no-cup propeller with the help of the cup trailing edge deformation.

Tab. 3. No-cup and cupped propeller section details

\begin{tabular}{|c|c|c|c|c|c|}
\hline $\mathbf{r} / \mathbf{R}$ & Chord / D & $\begin{array}{c}\mathbf{P} / \mathbf{D} \\
(\mathbf{N o}-\mathbf{C u p})\end{array}$ & $\begin{array}{c}\mathbf{P} / \mathbf{D} \\
(\mathbf{C u p p e d})\end{array}$ & $\mathbf{F}_{\max } / \mathbf{C}$ & $\mathbf{T}_{\max } / \mathbf{C}$ \\
\hline 0.24 & 0.349 & 1.296 & 1.056 & 0.021 & 0.124 \\
\hline 0.25 & 0.357 & 1.302 & 1.061 & 0.021 & 0.119 \\
\hline 0.30 & 0.380 & 1.320 & 1.075 & 0.022 & 0.101 \\
\hline 0.40 & 0.420 & 1.347 & 1.098 & 0.023 & 0.078 \\
\hline 0.50 & 0.449 & 1.365 & 1.112 & 0.024 & 0.063 \\
\hline 0.60 & 0.465 & 1.372 & 1.118 & 0.022 & 0.051 \\
\hline 0.70 & 0.466 & 1.369 & 1.116 & 0.018 & 0.041 \\
\hline 0.80 & 0.442 & 1.360 & 1.108 & 0.014 & 0.033 \\
\hline 0.90 & 0.373 & 1.344 & 1.095 & 0.010 & 0.026 \\
\hline 0.95 & 0.312 & 1.333 & 1.086 & 0.009 & 0.024 \\
\hline 0.98 & 0.245 & 1.326 & 1.080 & 0.008 & 0.025 \\
\hline 1.00 & 0.124 & 1.320 & 1.076 & 0.012 & 0.042 \\
\hline
\end{tabular}


The no-cup and cupped propeller 3D files are modeled. Fig. 5 shows the geometries of the propellers. The cup deflection can be observed on the trailing edge of the cupped propeller.
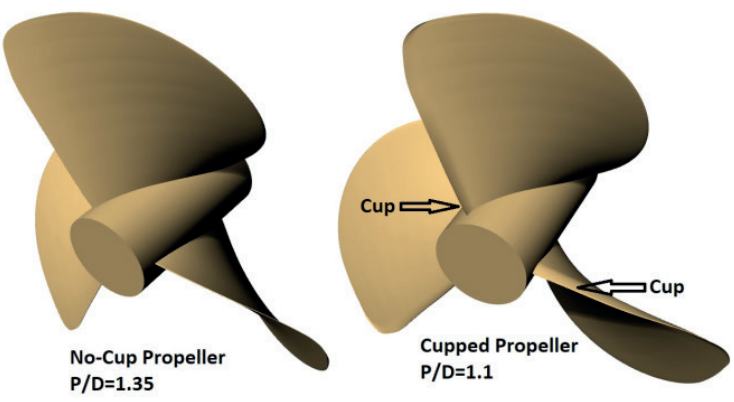

Fig. 5. No-cup propeller and cupped propeller view

In Fig. 6, the blade sections of the analyzed propellers are shown. The trailing edge deflection is defined by the manipulation of the camber function. The blade section of the cupped propeller indicates that the cup deflection starts from $0.95 \mathrm{C}$ of the section.

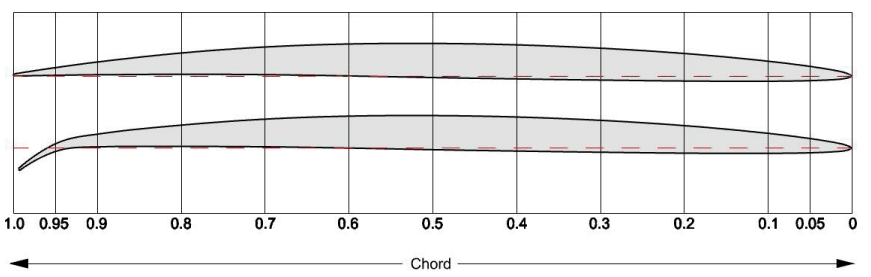

Fig. 6. No-cup and cupped propeller blade sections

The created cupped blade section has a lower angle of attack compared with the no-cup blade section. The reason for decreasing the angle of attack in the cupped propeller is to match the produced thrust with the no-cup propeller. The trailing edge deflection leads to a higher flow velocity on the trailing edge area and creates additional thrust [9]. However, the lower angle of attack reduces the peak velocity around the mid-chord. With the help of the cup and angle of attack optimizations, the cupped propeller is expected to produce the same open water results while creating a reduction in cavitation. The angle of attack difference of the propellers is shown in Fig. 7.
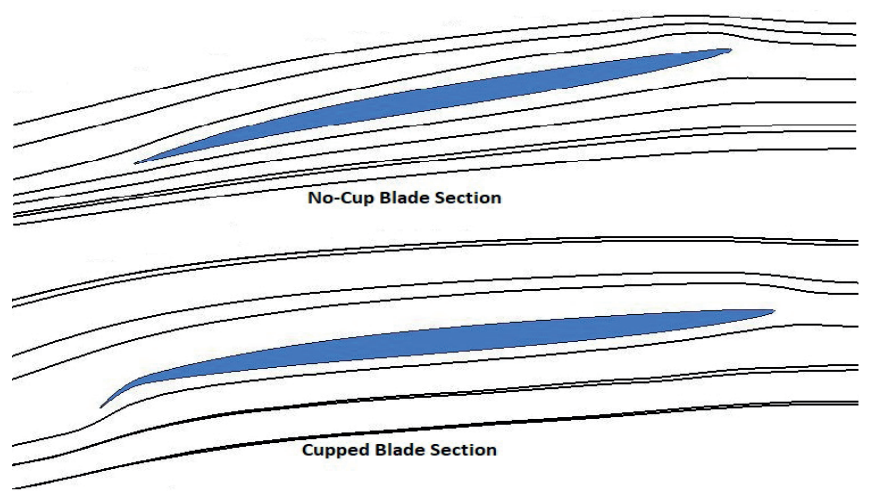

Fig. 7. Angle of attack comparison
Fig. 8 shows the computational domain dimension in terms of propeller diameter.

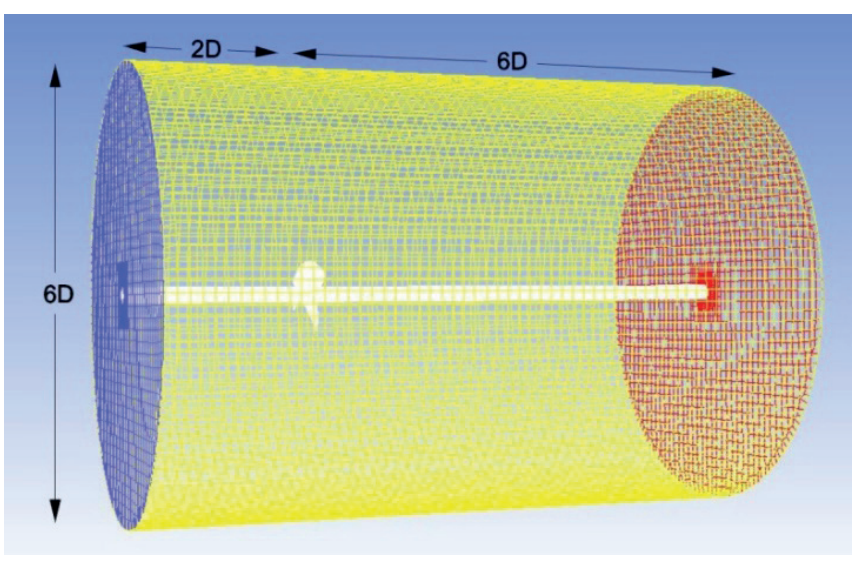

Fig. 8. Computational domain

The same calculation procedure and parameters are used in the analysis. The vaporization pressure is $3540 \mathrm{~Pa}$, which is the same for both the no-cup and cupped propeller calculation in order to compare the cavitation occurrence for the same depth conditions. The K- $\omega$ Shear Stress Transport (SST) turbulence model and Schnerr-Sauer cavitation model is used for the Reynolds Averaged Navier Stokes (RANS) calculations of both propellers [17] [18]. If the cavitation of a propeller is simulated using the RANS solver combined with the K- $\omega$ SST turbulence model and Schnerr-Sauer cavitation model, the behavior of the propeller cavitation is in good agreement with the test results [19]. CFD calculations are performed by using Ansys Fluent. The single-rotating-frame method is selected for the solutions. The first layer thickness of the viscous layer is $9 \times 10^{-5} \mathrm{~mm}$ and 10 layers are applied with a stretching ratio of 1.2. The $y+$ value is between $30-110$ on the major part of the blades, and $y+$ is reduced to 6 -12 approximately around the roots of the blades. A $10^{-8}$ residual error for continuity and $10^{-7}$ residual errors for $x-y-z$ velocity, k-omega, and vf-phase- 2 are achieved in the calculations.

Mesh sizes, which are defined as the surfaces of the propeller and shaft, are shown in Table 4 . The blade trailing edge cell size is reduced in order to create a fine mesh around the cupping area compared with the blade faces. The calculation domain has a full structured hexahedral mesh, which is created by Hexpress.

For the no-cup propeller mesh, the minimum orthogonal quality is 0.205 and the maximum skewness is 0.795 . For the cupped propeller mesh, the minimum orthogonal quality is 0.216 and the maximum skewness is 0.784 .

Tab. 4. Mesh parameters

\begin{tabular}{|c|c|c|}
\hline Mesh & No-Cupped & Cupped \\
\hline Blades & $3 \mathrm{~mm}$ & $3 \mathrm{~mm}$ \\
\hline Blade Trailing Edge & $0.3 \mathrm{~mm}$ & $0.3 \mathrm{~mm}$ \\
\hline Root & $2 \mathrm{~mm}$ & $2 \mathrm{~mm}$ \\
\hline Shaft & $5 \mathrm{~mm}$ & $5 \mathrm{~mm}$ \\
\hline Total Cell & 7547218 & 7672828 \\
\hline
\end{tabular}


Fig. 9 shows the surface mesh view of the propeller. As shown on the mesh parameters table, the trailing edge mesh is finer.

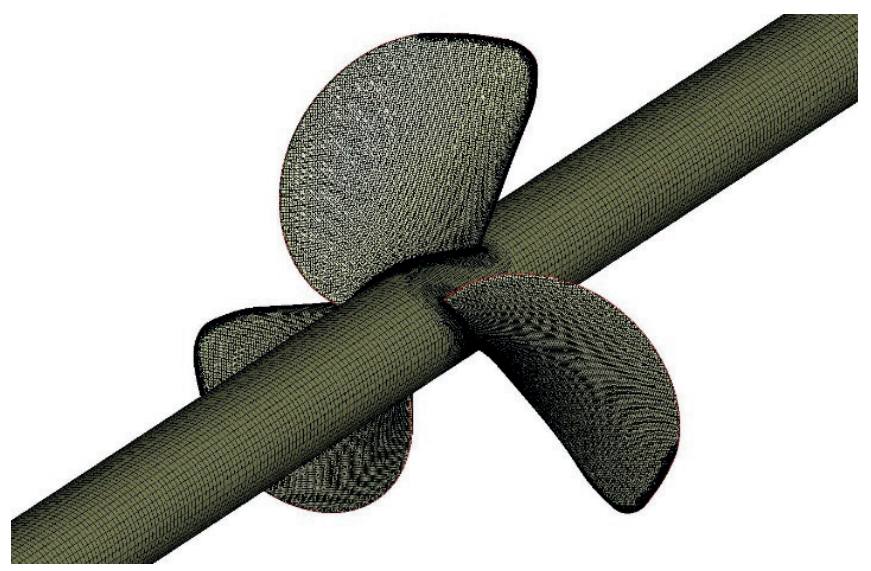

Fig. 9. Cupped propeller cells on suction side

The calculations are performed as steady. The analyses are solved without the vapor phase first. In this initial analyses, which provides a converged start to cavitation calculations, Simplec segregated solver is used. Discretization schemes are least-square cell-based for the gradient and second-order for pressure, momentum, turbulent kinetic energy, and specific dissipation rate. After convergence is satisfied to $10^{-7}$ residual error, the multiphase model is solved with Simplec solver. The same discretization schemes are used but Presto for pressure and Quick for volume fraction is selected additionally.

\section{RESULTS AND DISCUSSION}

Two different propellers that are working in two different advance coefficients are solved with CFD. The calculation results are shown in Fig. 10 as open water curves. The $\mathrm{K}_{\mathrm{T}}, \mathrm{K}_{\mathrm{O}}$, and efficiency results indicate that one of the main goals is satisfied. The first main goal is creating a cupped propeller that is producing the same thrust that can be replaced by the no-cup propeller without any additional revisions or replacements on the propulsion components of the boat.

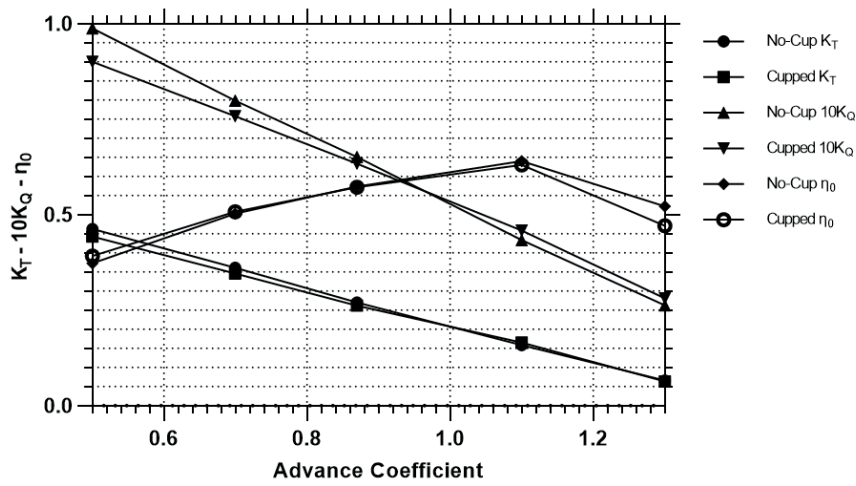

Fig. 10. Open water curves of no-cup and cupped propellers
$K_{T}=\frac{T}{\rho n^{2} D^{4}}, \quad K_{Q}=\frac{Q}{\rho n^{2} D^{5}}, \quad \eta_{0}=\frac{J K_{T}}{2 \pi K_{Q}}$

Table 5 shows that the efficiency values of the defined propellers are almost the same for the advance coefficients where the cavitation comparison is performed.

Tab. 5. Propeller efficiency comparison

\begin{tabular}{|c|c|c|}
\hline J & No-Cupped & Cupped \\
\hline 0.82 & $55.1 \%$ & $56.6 \%$ \\
\hline 0.87 & $56.7 \%$ & $56.5 \%$ \\
\hline
\end{tabular}

The cavity patterns of the no-cup propeller for the 0.82 and 0.87 advance coefficients, which are created by the lifting surface, are shown in Fig. 11. The cavity patterns are expected to be in agreement with the CFD cavitation plots.

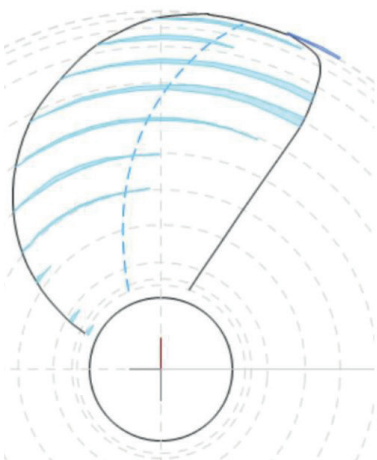

No-Cup Propeller $\mathrm{J}=0.82$

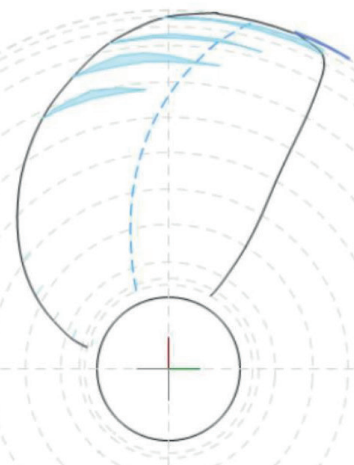

No-Cup Propeller $\mathrm{J}=0.87$
Fig. 11. Cavitation patterns of no-cup propeller at 0.82 and 0.87 advance coefficients by the lifting surface method

The second goal is to observe a reduction in cavitation. Figs. 12 and 13 show the vapor fraction comparison of the propellers at advance coefficients 0.82 and 0.87 . For $\mathrm{J}=0.82$, cavitation risk is observed starting from $0.5 \mathrm{R}$ of the blade to the tip (left). However, on the cupped version of the specified propeller (right), only the trailing edge and the tip of the blade generate cavitation risk. The agreement between the lifting surface method and CFD can be noticed for the no-cup results [20].

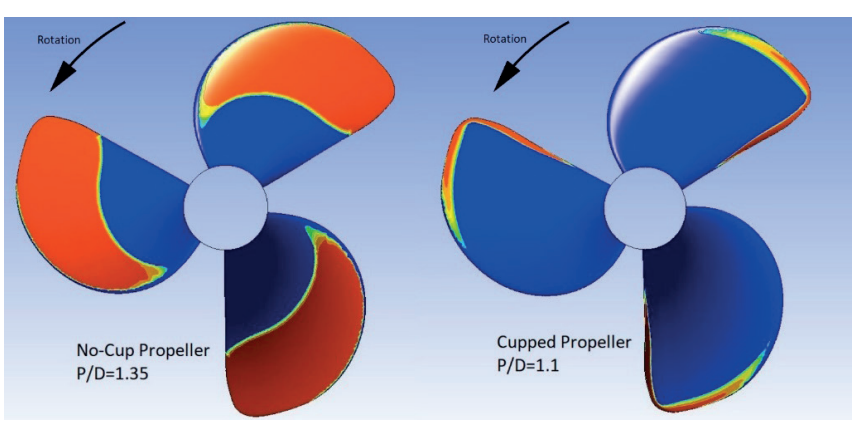

Fig. 12. Vapor fraction comparison at $J=0.82$ 
Compared with the 0.82 advance coefficient, 0.87 has a lower cavitation-producing potential because of the lower local velocities on the blades. Even having a lower rotational speed and inlet velocity at 0.87 , a vapor fraction over 0.5 can be observed from $0.7 \mathrm{R}$ to the tip (left). However, the cupped version propeller (right) almost eliminated the cavitation occurrence. The phase change is found only on the trailing edge area between the $0.7 \mathrm{R}$ and tip, as shown in Fig. 13 .

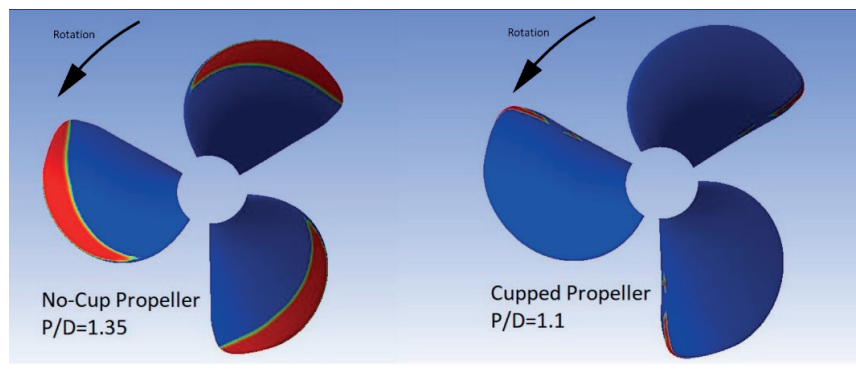

Fig. 13. Vapor fraction comparison at $J=0.87$

The iso-surface plot at vapor fraction 0.5 is shown in Fig. 14 at advance coefficient 0.87 . The cupped version of the propeller, which has a lower blade angle of attack, creates a better cavitation characteristic at the defined advance coefficient.

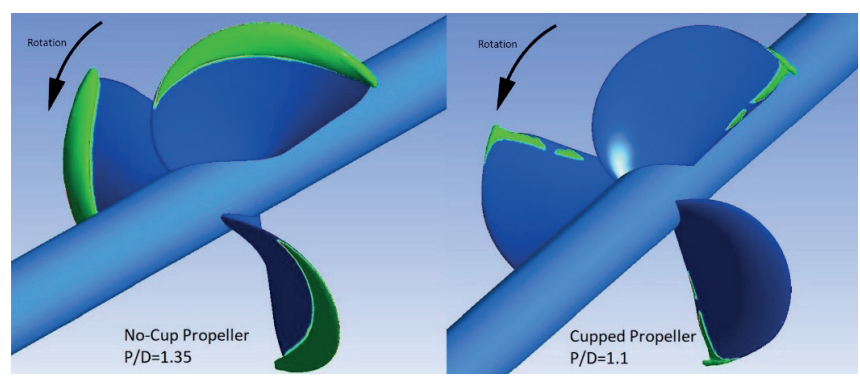

Fig. 14. 0.5 vapor fraction comparison at $J=0.87$

Figs. 15 and 16 show the pressure coefficient plots of the propellers at advance coefficients 0.82 and 0.87 . If the pressure coefficient plots are checked according to the defined $\sigma$ values in the figures, the agreement in the vapor fraction plots and pressure coefficient can be observed.
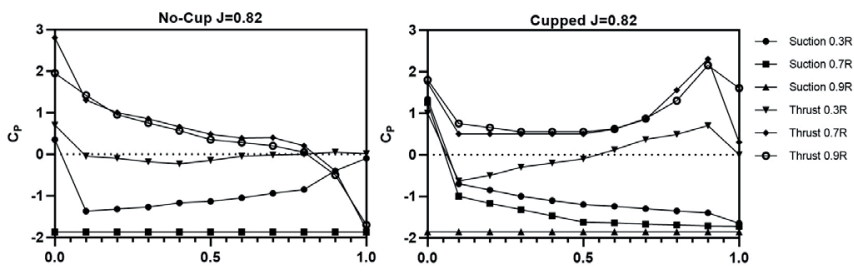

Fig. 15. Pressure coefficient distribution at $-\sigma=1.85$
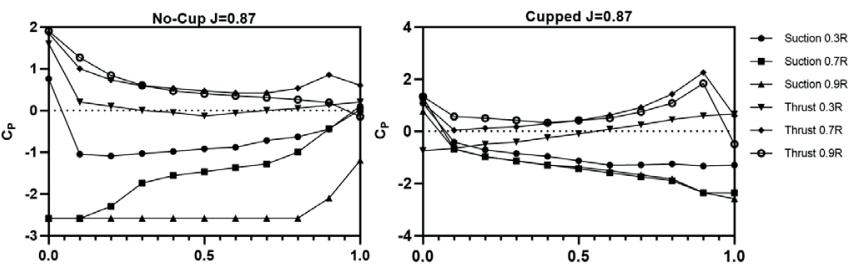

Fig. 16. Pressure coefficient distribution at $-\sigma=2.59$

\section{CONCLUSION}

Erosion is a crucial issue for marine propellers. In order to provide a long life-time, cavitation elimination is fundamental. As detailed in the previous sections, there are several methods for cavitation reduction on the blades. However, all of these mentioned methods for cavitation reduction are able to be applied at the initial stages of the design of the boat.

Hull speed, hull stern shape, propeller-hull clearance, engine power, and gearbox reduction ratio should be correctly examined. However, for the specific cases where there are solid design restrictions, modifying the pressure distribution on the blade by propeller cup could be an alternative cavitation elimination method. By virtue of the propeller cup, the angle of attack of the blades is reduced after determining the cavitation distribution of the conventional propeller. A well-optimized blade cup compensates for the lost thrust that comes from the blade angle of attack decrement.

The total pressure difference between the low-pressure side and the high-pressure side of the propeller is kept constant. The critical point of the cup determination is to examine the influence of the different cup levels on the angle of attack alteration of the blades. Several CFD calculations should be performed for a range of $\mathrm{P} / \mathrm{D}$ values to adjust the pitch of the cupped version of the studied cavitating propeller.

The thrust and torque coefficients and efficiency comparison tables for the specified cases show that a no-cup propeller can be converted to a cupped propeller that provides the same performance characteristics and has the same diameter and rotational speed but a different angle of attack.

Vapor fraction and cavitation coefficient plots indicate a significant reduction in the cavitation occurrence on the blades, which are found for two different advance coefficients. Determination of the cup level and P/D modification are the main objectives and also the main challenges. The $\mathrm{P}_{\mathrm{EFF}}=\mathrm{P}_{\mathrm{GEO}}+21\left(\mathrm{X}_{\mathrm{CUP}}\right)$ formulation provides a solid starting point and offers an opportunity to identify a range for $\mathrm{P} / \mathrm{D}$ modification [8] [10].

CFD calculations should be performed for the final tuning of the modified (cupped) propeller. Thrust, torque, and efficiency values should be examined attentively as well as the cavitation results. In order to create a systematic cup creation method, the blade pitch conversion formulation should be tested with CFD calculations for a P/D range, which should be the focus of future work. 


\section{REFERENCES}

1. T. Koronowicz, Z. Krzemianowski, T. Tuszkowska, J.A. Szantyr, "A Complete Design of Ship Propellers Using New Computer System” Polish Maritime Research 1(59), vol. 16, pp. 29-34, 2009.

2. T. Koronowicz, Z. Krzemianowski, "Investigation of Influence of Screw Propeller Operation on Water Flow Around Stern Part of Ship Hull" Polish Maritime Research 1(51), vol 14, pp. 3-8, 2007.

3. A. Peters, U. Lantermann, O. el Moctar, "Numarical Prediction of Cavitation Erosion on a Ship Propeller in Model- and full-scale" Wear, vol. 408-409, pp. 1-12, 15 August 2018.

4. B. Aktas, M. Atlar, S. Turkmen, W. Shi, R. Sampson, E. Korkut, P. Fitzsimmons, "Propeller Cavitation Noise Investigations of a Research Vessel Using Medium Size Cavitation Tunnel Tests and Full-Scale Trials" Ocean Engineering, vol. 120, pp. 122-135, 1 July 2016.

5. J.W. Lindau, D.A. Boger, R.B. Medvitz, R.F. Kunz, "Propeller Cavitation Breakdown Analysis" Journal of Fluids Engineering, vol. 127(5), pp. 995-1002, Sep 2005.

6. J. G. Peck, B. L. Fisher, "Cavitation Performance of Propellers With and Without Cupping” David W. Taylor Naval Ship Research and Development Center, Sep 1976

7. K. W. Shin, P. Andersen "CFD Analysis of Cloud Cavitation on Three Tip-Modified Propellers With Systematically Varied Tip Geometry" J. Phys. :Conf. Ser 656 012139, 2015.

8. Donald M. MacPherson, HydroComp Technical Report, Small Propeller Cup, Presented at the Propeller/Shafting Symposium, 1997

9. Jing-Fa Tsai, "Study on the Cavitation Characteristics of Cupped Foils" Journal of Marine Science and Technology, vol. 2, pp. 123-134, 1997.

10. Hwang, J. L, Tsai, J: F., Li C. Y., "Cupped Propeller Test and Analysis”, vol. 42, issue 4, pp. 186-192, October 1995

11. HydroComp Technical Report, Correlating Michigan Wheel Cup Gages

12. E. Korkut, M. Atlar, "An Experimental Study into the Effect of Foul Release Coating on the Efficiency, Noise and Cavitation Characteristics of a Propeller" First International Symposium on Marine Propulsors smp'09, Trondheim, Norway, June 2009
13. E. V. Lewis, Principles of Naval Architecture, Volume II, Resistance, Propulsion, and Vibration, Society of Naval Architects and Marine Engineers, Jersey City, NJ, 1988.

14. E. C. Tupper, "Introduction to Naval Architecture", Fifth Edition, 2013

15. J. Carlton, 2007, "Marine Propellers and Propulsion", Second Edition, Butterworth-Heinemann

16. P. Krol, "Blade Section Profile Array Lifting Surface Design Method for Marine Screw Propeller Blade" Polish Maritime Research 4 (104), vol. 26, pp. 134-141, 2019.

17. D.-C. Liu, W.-X. Zhou, "Numerical Predictions of the Propeller Cavitation Pressure Fluctuation behind Ship and Comparison with Experiment", Journal of Ship Mechanics, vol. 23(3), pp. 245-254, March 2019.

18. N. Lu, G. Bark, U. Svennberg, R. Bensow, "Numerical Simulations of the Cavitating Flow on a Marine Propeller", Proceedings of the Eighth International Symposium on Cavitation, pp. 338-343, 2012.

19. L.Guangnian, Q. Chen, Y. Liu "Experimental Study on Dynamic Structure of Propeller Tip Vortex" Polish Maritime Research 2 (106), vol. 27, pp. 11-18, 2020.

20. S. Sezen, A. Dogrul, S. Bal, “An Empirical Approach For Propeller Tip Vortex Cavitation Noise”, Sigma J Eng \& Nat Sci, vol. 36 (4), pp. 1127-1139, 2018.

\section{CONTACT WITH THE AUTHOR}

\author{
M. Burak Samsul \\ e-mail:samsul@itu.edu.tr \\ Istanbul Technical University, \\ Maslak, 34467 Istanbul
}

TÜRKIYE 